\title{
Management of meconium-stained amniotic fluid (MSAF) and the meconium aspiration syndrome (MAS)
}

\author{
G N Lucas ${ }^{1}$ \\ Sri Lanka Journal of Child Health, 2002; 31: 118-21
}

(Key words: meconium stained amniotic fluid, meconium aspiration syndrome, MSAF, MAS)

\section{Introduction}

Meconium is a viscous green fluid consisting of gastrointestinal secretions, bile, mucus, pancreatic juice, cellular debris, amniotic fluid, swallowed vernix caseosa, lanugo and blood ${ }^{1}$. In utero passage of meconium is uncommon due to the relative lack of strong peristalsis, good anal sphincter tone and a "cap" of particularly viscous meconium in the rectum ${ }^{1}$.

\section{The scope of the problem}

The overall frequency of meconium-stained amniotic fluid (MSAF) has ranged from 5.6-24.6\% (median $14 \%$ ) of all deliveries ${ }^{2}$. In 2 studies done at the Castle Street Hospital for Women (CSHW) the frequencies were $4 \%$ and $6 \%$ respectively ${ }^{3.4}$. Of those born through MSAF, 1.7-35.8\% (median 10.5\%) have developed meconium aspiration syndrome (MAS) ${ }^{2}$. In the 2 studies at CSHW 31\% and 34\% babies with MSAF respectively developed $\mathrm{MAS}^{3.4}$. Death has occurred in 4.9-37\% (median 12\%) infants with MAS2. In the 2 studies at CSHW, death occurred in $14.2 \%$ and $7.3 \%$ infants with MAS respectively ${ }^{3.4}$.

Passage of meconium has been taken as a marker of antepartum or intrapartum asphyxia. The hypothesis is that in utero hypoxia causes increased intestinal peristalsis and relaxed anal sphincter tone, resulting in meconium passage ${ }^{5}$. In addition, compression of the fetal head or umbilical cord may cause a vagal response and meconium passage ${ }^{5}$. In the 2 studies at CSHW $47 \%$ and $20 \%$ babies with MSAF were asphyxiated at birth ${ }^{3,4}$. For most infants, passage of meconium represents a maturational event. Meconium passage is rare before 37 weeks gestation but may occur in $35 \%$ or more pregnancies lasting longer than 42 weeks $^{6}$. In the 2 studies at CSHW $30 \%$ and $2 \%$ babies with MSAF were post-term and $0 \%$ and $1 \%$ were preterm ${ }^{3,4}$.

${ }^{1}$ Consultant Paediatrician \& Joint Editor

\section{Definition of MAS}

MAS is defined as respiratory distress in an infant born through MSAF whose symptoms cannot be otherwise explained ${ }^{2}$. As there are a wide variety of $\mathrm{x}$-ray findings among infants with MAS, no specific radiographic features are used as part of the diagnosis $^{2}$.

\section{Pathophysiology of MAS ${ }^{2}$}

Factors involved in the complex pathophysiology of MAS include

- Airway obstruction caused by meconium, oedema fluid, protein exudate, red and white blood cells.

- Alveolar and parenchymal inflammation.

- Protein leak into the airways.

- Effect of mediators - cytokines, eicosanoids.

- Surfactant dysfunction caused by meconium, protein and inflammatory cells.

- Direct toxicity of meconium constituents.

- Vasoconstriction from components of meconium.

- $\quad$ Altered pulmonary vasoreactivity.

- Effects of in utero hypoxaemia.

\section{Radiographic findings of $\mathrm{MAS}^{2}$}

Although classic x-ray findings of MAS are described as diffuse, patchy infiltrates, diverse mechanisms involved in its pathogenesis lead to a wide variety of radiographic findings. These include consolidation, atelectasis, pleural effusions, air leaks (e.g. pneumothorax or pneumomediastinum), hyperinflation, a wet-lung picture, hypovascularity or a relatively normal appearance. 


\section{Meconium aspiration: Does it occur in utero or after birth?}

It is likely that meconium aspiration occurs from both in utero gasping and postpartum aspiration with the initial breaths of the baby. It is impossible to determine the proportion of cases of MAS that are caused by each mechanism. Among individual neonates with MAS there is no way of distinguishing which particular mechanism of aspiration occurred ${ }^{2}$.

MAS: Is the pathology primarily due to asphyxia and not the meconium?

The classic postmortem findings of MAS are obstructive phenomena-atelectasis alternating with overexpanded parenchyma as well as pulmonary air leaks ${ }^{7}$. The common x-ray findings of areas of atelectasis interspersed with hyperexpanded regions are consistent with direct obstruction by meconium itself ${ }^{2}$. Furthermore, in reviewing the literature, Wiswell et al found a large proportion of infants with MAS (30-56\%) to have been vigorous and apparently healthy at birth ${ }^{8}$.

\section{Efficacy of obstetric and paediatric interventions}

Katz and Bowes question the efficacy of obstetric hypopharyngeal suctioning as well as neonatal intratracheal suctioning, in preventing or alleviating the severity of $\mathrm{MAS}^{9}$. However, the studies of Gregory, Ting, Carson and their colleagues all demonstrate the benefits of aggressive airway management in meconium-stained neonates ${ }^{10.11 .12}$. Furthermore, in a large comprehensive review of more than 21,000 meconium-stained infants born over a 15 year period, Wiswell et al found aggressive airway management to result in significantly less MAS and deaths due to $\mathrm{MAS}^{8}$

\section{Universal versus selective intratracheal suctioning}

Cunningham and colleagues called for a selective approach to intubation of the meconium-stained infant. These authors contended that laryngoscopy and tracheal suctioning should be reserved for infants who are depressed and require positive pressure ventilation in the delivery rooml ${ }^{3}$. On the other hand, it should be realised that a meconium-stained neonate who is apparently healthy can still develop MAS ${ }^{2}$. Furthermore, of those infants who developed MAS, 7.3-35\% (median $17 \%$ ) had been born through thin-consistency MSAF $^{2}$. Potential but rare complications of routine intubation in the delivery room include prolonged reflex bradycardia, perforation of the pharynx, transient or persistent hoarseness/stridor and laryngospasm ${ }^{1}$. The current joint guidelines of the American Academy of Paediatrics and American Heart Association recommend intubation of all infants born through moderately thick or thick- consistency MSAF. Infants born through thinconsistency meconium may not need intubation if adequate oropharyngeal suctioning is performed and if the infant is vigorous and does not need positivepressure ventilation $^{14}$.

\section{Pharyngeal suction by the obstetrician}

Carson et al was the first to document the beneficial effect of pharyngeal suctioning prior to delivery of the infant's shoulders ${ }^{12}$. Nevertheless, even with such suctioning $21-37 \%$ of infants will still have meconium present in their tracheae. Furthermore, infants still develop MAS despite optimal pharyngeal and intratracheal cleansing ${ }^{15}$. Although pharyngeal suctioning has played a major role in the declining incidence of MAS it is not a panacea $^{8}$.

\section{Saline lavage}

As part of airway toilet with meconium below the vocal cords, perhaps saline lavage would be of benefit. This therapy could "thin" tenacious meconium and assist in its removal. Beeram and Dhanireddy evaluated pulmonary mechanics in 9 ventilated neonates with MAS following intratracheal saline instillation. They found a sustained decrease in airway resistance after saline administration and tracheal suction ${ }^{16}$.

\section{Amnioinfusion}

Initial reports from the late 1980 s and early 1990 s indicated that amnioinfusion for thick consistency MSAF would improve Apgar scores and prevent MAS ${ }^{1}$. With this technique, fluid (generally, warmed normal saline) is infused into the uterus of mothers whose fetuses are thought to be "at risk". For the meconiumstained infant, it is believed that increasing the uterine fluid volume may either dilute the thickness of the meconium (and reduce its toxicity) or alleviate cord compression (and prevent gasping). Unfortunately, more recent reports have not found this therapy to be of benefit $^{17}$. Moreover, use of amnioinfusion has been associated with significantly more fetal heart rate abnormalities, increased rates of caesarean section and instrument-assisted deliveries and increased rates of neonatal sepsis ${ }^{18}$.

\section{Recommended delivery room management of meconium-stained infant}

Taking all the above matters into consideration the following combined obstetric-paediatric approach is recommended. This is diagrammatically represented in Figure 1. 


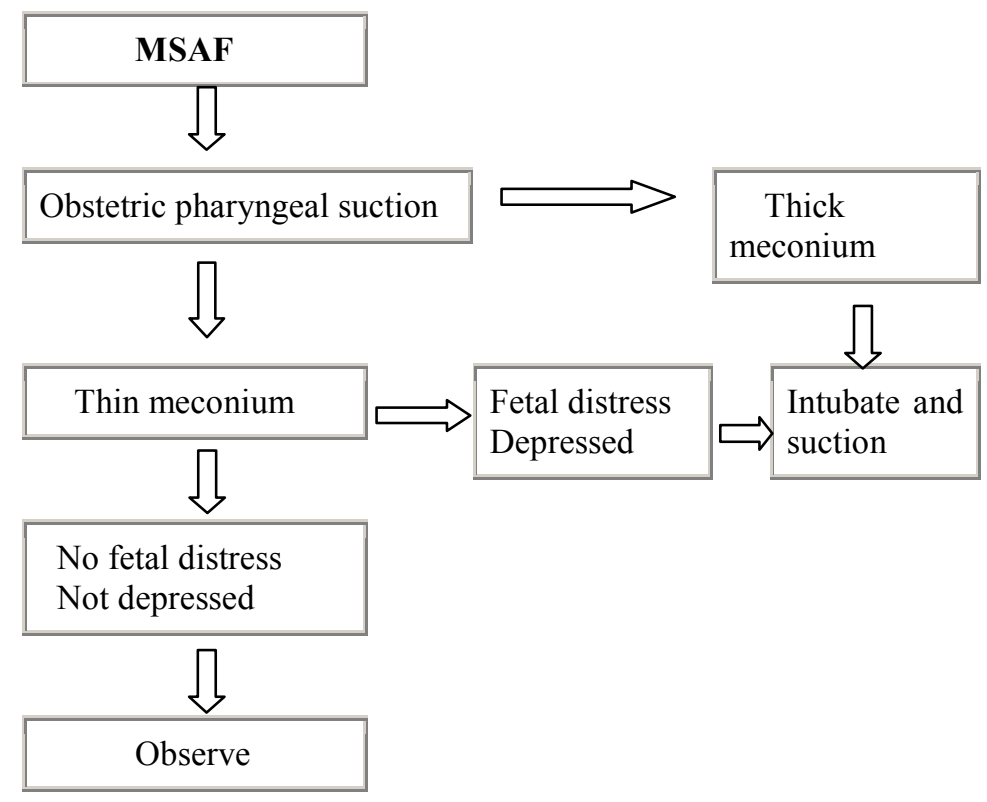

Figure 1. Delivery room management protocol

- As soon as the baby's head is delivered (prior to delivery of the shoulders) the mouth, oropharynx and hypopharynx should be thoroughly suctioned using a flexible suction catheter no smaller than a $10 \mathrm{Fr}$.

- After delivery of the infant, the trachea should be intubated and any residual meconium removed from the lower airway. In the presence of thick meconium this is best done by applying suction to an endotracheal tube. Once the endotracheal tube has been inserted, continuous suction is applied to the tube as it is withdrawn. Reintubation followed by suctioning should be repeated until returns are free of meconium. Attempts should not be made to suction meconium from the trachea by passing a suction catheter through an endotracheal tube as the catheter size required to fit through an endotracheal tube in a newborn is too small to adequately remove meconium.

- It is difficult and potentially hazardous to attempt endotracheal intubation in a conscious struggling newborn. In such cases it is preferable to suck out the meconium from the pharynx and larynx under direct vision with the aid of a laryngoscope.

\section{Management of MAS}

\section{Antibiotics}

Even though there is little evidence to justify the routine use of antibiotics for MAS, it is not easy to distinguish the early radiographic picture of the disorder from that of neonatal pneumonia. Thus it is recommended that antibiotics are given to infants with MAS; however, such therapy should be discontinued as soon as cultures have been negative for 48-72 hours. Generally, a combination of penicillin and gentamicin are used.

\section{Chest physiotherapy (CPT)}

The objectives of CPT are to prevent accumulation of debris and improve mobilization of airway secretions and improve the efficiency and delivery of oxygenation ${ }^{19}$. CPT consists of several discrete techniques: postural drainage, percussion, vibration, oropharyngeal and tracheal suctioning. There are scant data documenting the efficacy of CPT use in MAS.

\section{Conventional mechanical ventilation}

One third of infants with MAS require mechanical ventilation $^{8}$. Because air leaks are a common complication of MAS, particularly among infants requiring positive pressure ventilation, perhaps we should try to use oxygen alone (up to an $\mathrm{FiO}_{2}$ of 1.00) longer than we typically do for other respiratory disorders (presuming hypercapnoea and acidosis are not major problems) $)^{2}$. 
Once a child requires intermittent mandatory ventilation, what are the best ventilator settings? There are advocates of low inspiratory pressures, short inspiratory times and rapid ventilator rates to be used to maintain arterial blood gases within normal limits. There have been no published studies comparing this strategy with more commonly used ventilator settings.

Because two thirds of the neonates with persistent pulmonary hypertension of the newborn (PPHN) have associated MAS, hyperventilation is a common approach in the management of MAS with the goal of attaining respiratory alkalosis in an effort to achieve pulmonary vasodilation.

\section{High-frequency ventilation}

High-frequency ventilation (HFV) describes several techniques which provide effective gas exchange at low tidal volumes ${ }^{20}$. Potential advantages of HFV in treating MAS include less barotrauma, increased mobilisation of airway secretions, ease of attaining respiratory alkalosis and fewer adverse histopathologic changes.

\section{Extracorporeal membrane oxygenation (ECMO) in MAS}

ECMO is a form of cardiopulmonary bypass which is generally used when infants are failing more conventional management and have a higher risk of dying compared with historical controls ${ }^{21}$. The majority of infants with MAS placed on ECMO manifest PPHN. They are placed on bypass support in the hope their lung disease will improve over a period of days. The survival rate of these children is generally $85 \%$ or higher. The technique is not benign, however, and many complications have been described (chiefly haemorrhagic sequelae due to anticoagulation

therapy).

\section{Inhaled nitric oxide in MAS}

Inhaled nitric oxide (INO) has been successfully used in the treatment of neonates with PPHN several of whom had $\mathrm{MAS}^{22}$. There was a prompt improvement in oxygenation without systemic hypotension following nitric oxide administration.

\section{Systemic steroid therapy}

Inflammation plays a major role in the pathophysiology of MAS. Glucocorticoids have been used for the treatment of chemical pneumonitis and could potentially benefit MAS. Yeh and colleagues prospectively examined hydrocortisone therapy in a group of 35 infants with MAS. They did not find any differences in blood gases or the needed degree of respiratory support ${ }^{23}$.

\section{Surfactant therapy}

Meconium has been shown to inhibit surfactant in vitro. This inhibition appears to depend on the concentration of surfactant, suggesting the possible benefits of exogenous surfactant therapy in managing MAS. To date, there has been only one randomized, controlled trial specifically assessing the use of exogenous surfactant therapy for $\mathrm{MAS}^{24}$. In this study, 20 affected infants were treated with 1.5 times the standard dose of a bovine lung surfactant which was administered as an infusion over 20 minutes. Significant improvements in oxygenation occurred 612 hours later, typically following additional surfactant doses. The authors recommend further clinical trials before widespread use of this therapy for MAS.

\section{References}

1. Wiswell T E, Brent R C. Meconium staining and the meconium aspiration syndrome: Unresolved issues. Paediatric Clinics of North America 1993; 40: $955-81$.

2. Cleary G M, Wiswell $\mathrm{T}$ E. Meconium stained amniotic fluid and the meconium aspiration syndrome: An update. Paediatric Clinics of North America 1998; 45: 511-29.

3. Lucas G N, Dissanayake P. A study of meconium staining and meconium aspiration syndrome in a maternity hospital. Ceylon Medical Journal 1995; 40: $62-3$.

4. Lucas G N, Rankothge M. Meconium aspiration syndrome at the Castle Street Hospital. Ceylon Journal of Child Health 1995; 24: 35-6.

5. Miller F C, Read J A. Intrapartum assessment of the postdate fetus. American Journal of Obstetrics and Gynecology 1981; 141: 516.

6. Eden R D, Seifert L S, Winegar A. Perinatal characteristics of uncomplicated postdate pregnancies. Obstetrics and Gynecology 1987; 151: 731.

7. Davis R O, Philips J B, Harris B A. Fatal meconium aspiration syndrome occurring despite airway management considered appropriate. American Journal of Obstetrics and Gynecology1985; 151: 731. 
8. Wiswell $\mathrm{T}$ E, Tuggle $\mathrm{J}$ M, Turner $\mathrm{B}$ S. Meconium aspiration syndrome: Have we made a difference? Pediatrics 1990; 85: 715.

9. Katz V L, Bowes W A. Meconium aspiration syndrome: Reflections on a murky subject. American Journal of Obstetrics and Gynecology 1992; 166: 171.

10. Gregory G A, Gooding C A, Phibbs R H. Meconium aspiration in infants -a prospective study. Journal of Pediatrics 1974; 85: 848.

11. Ting $\mathrm{P}$, Brady $\mathrm{J}$ P. Tracheal suction in meconium aspiration. American Journal of Obstetrics and Gynecology 1975; 122:767.

12. Carson B S, Losey R W, Bowes W A. Combined obstetric and paediatric approach to prevent meconium aspiration syndrome. American Journal of Obstetrics and Gynecology 1976; 126: 712 .

13. Cunningham A S, Lawson E E, Martin R J. Tracheal suction and meconium: A proposed standard of care. Journal of Pediatrics 1990; 116: 153 .

14. Committee on neonatal ventilation/meconium /chest compressions. Guidelines proposed at the 1992 National Conference on Cardiopulmonary Resuscitation and Emergency Cardiac Care, Dallas, 1992. Journal of the American Medical Association 1992; 268: 2276.

15. Hageman J R, Conley M, Francis K. Delivery room management of meconium staining of the amniotic fluid and the development of meconium aspiration syndrome. Journal of Perinatology1988; 8: 127.
16. Beeram M R, Dhanireddy R. Effects of saline instillation during tracheal suction on lung mechanics in newborn infants. Journal of Perinatology 1992; 12: 120.

17. Lo K W K, Rogers M. A controlled trial of amnioinfusion: The prevention of meconium aspiration in labour. Australia New Zealand Obstetrics and Gynaecology 1993; 33: 51.

18. Rogers $\mathrm{M} \mathrm{S}$, Lau $\mathrm{T} \mathrm{K}$, Wang $\mathrm{C}$ C. Amnioinfusion for the prevention of meconium aspiration during labour. Australia New Zealand Obstetrics and Gynaecology 1996; 36: 407.

19. Turner B S. Maintaining the artificial airway: Current concepts. Paediatric Nursing 1990; 16: 487.

20. Froese A B, Bryan C. High frequency ventilation. American Review of Respiratory Diseases, 1987; 135: 1363.

21. Short B L, Miller M K, Anderson K O. ECMO in the management of respiratory failure in the newborn. Clinical Perinatology1987; 14: 737.

22. Roberts J D, Polaner D M, Lang P. Inhaled nitric oxide in persistent pulmonary hypertension of the newborn. Lancet 1992; 340:818.

23. Yeh T F, Srinivasan G, Harris V. Hydrocortisone therapy in meconium aspiration syndrome: A controlled study. Journal of Paediatrics 1977; 90: 140 .

24. Findlay $R$ D, Taeusch $H$ W, Walther $F$ J. Surfactant replacement therapy for meconium aspiration syndrome. Pediatrics 1996; 97: 48. 
